\section{Florent Jacquemard Michael Rusinowitch}

Closure of Hedge-Automata Languages by Hedge Rewriting

Research Report LSV-08-05

February 2008

Laboratoire

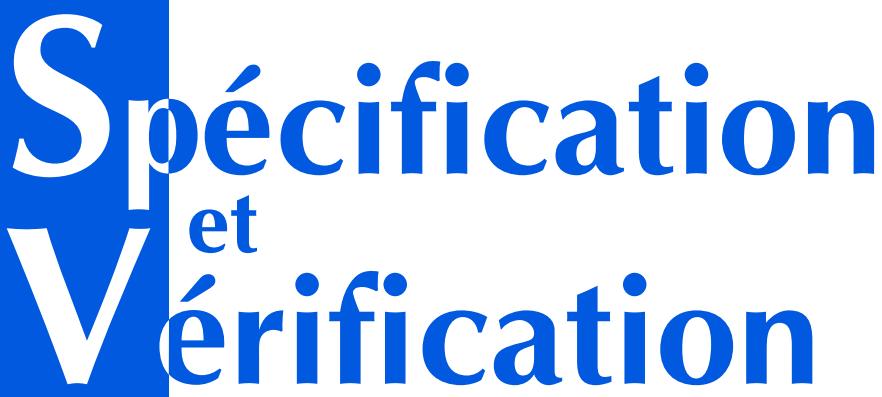

Ecole Normale Supérieure de Cachan 



\title{
Closure of Hedge-Automata Languages by Hedge Rewriting
}

\author{
Florent Jacquemard ${ }^{1}$ and Michael Rusinowitch ${ }^{2}$ \\ 1 INRIA Futurs \& LSV, UMR CNRS, ENS Cachan, France \\ florent.jacquemard@lsv.ens-cachan.fr \\ 2 LORIA \& INRIA Lorraine, UMR 7503, rusi@loria.fr
}

\begin{abstract}
We consider rewriting systems for unranked ordered terms, i.e. trees where the number of successors of a node is not determined by its label, and is not a priori bounded. The rewriting systems are defined such that variables in the rewrite rules can be substituted by hedges (sequences of terms) instead of just terms. Consequently, this notion of rewriting subsumes both standard term rewriting and word rewriting. We investigate some preservation properties for two classes of languages of unranked ordered terms under this generalization of term rewriting. The considered classes include languages of hedge automata (HA) and some extension (called CF-HA) with context-free languages in transitions, instead of regular languages.

In particular, we show that the set of unranked terms reachable from a given HA language, using a so called inverse context-free rewrite system, is a HA language. The proof, based on a HA completion procedure, reuses and combines known techniques with non-trivial adaptations. Moreover, we prove, with different techniques, that the closure of CF-HA languages with respect to restricted context-free rewrite systems, the symmetric case of the above rewrite systems, is a CF-HA language. As a consequence, the problems of ground reachability and regular hedge model checking are decidable in both cases. We give several counter examples showing that we cannot relax the restrictions.
\end{abstract}

\section{Introduction}

In many applications the system states can be modeled by words or trees, sets of configurations by word or tree languages and the transitions of the system can be represented by rewrite rules. In this setting verifying whether a system can enter a set of unsafe states can be expressed as a reachability problem. This approach to the analysis of infinite-state systems requires the computation of the closure of languages under rewrite rules or at least an over-approximation of this closure. Since the usually considered languages are regular the approach is called regular model checking $[2,1]$. Regular model checking has been quite successful in protocol and hardware verification. For increasing the scope of regular model checking it is therefore important to be able to derive new classes of languages and rewrite systems such that the rewrite closure is computable. 
Unranked trees as well as ordered sequences of unranked trees called hedges $[13,14,5]$ are flexible structures that are quite appealing to represent XML documents where the number of nodes can be modified, for instance when these nodes correspond to database records. Unranked trees have also been employed to model multithreaded recursive program configurations where the number of parallel processes is unbounded [3,18]. Hedge-automata (HA) are considered now as the natural model of automata for unranked trees. A hedge automaton is a variation of tree automata for hedges. Given a hedge, a hedge automaton assigns some state to a node whenever the sequence of states of the siblings belong to some specified word language (sometimes called horizontal language).

Although regular model checking with languages for words and ranked trees (where function symbols have fixed arity) has been widely investigated, very few results are available for unranked trees and almost none exists on the computation of exact reachability sets for HA languages.

In this paper we tackle the problem above by proving (Theorem 1) that we can compute a HA for recognizing the rewrite closure of a language defined by a given HA, for the class of rewrite systems with inverse context-free rules, which are rules whose right-hand side is of type $f(x)$ where $x$ is a variable. Hence in that case we can compute the exact reachability set from the initial one. The rewriting notion that we consider here for unranked terms generalizes ranked term rewriting and is close to the one that has been introduced by [22]. The idea is that the variables in the rewrite rules can be substituted by hedges (sequences of terms) instead of just terms. Moreover our results cannot be derived from related ones on ranked terms (e.g. [15]) using encodings of unranked terms into ranked ones (such as the First-Child-Next-Sibling encoding or the encoding used in stepwise automata [4]). Relaxing the condition in the definition in the above class of rewrite systems leads to counterexamples (Propositions 3-6).

We have also considered a more general class of automata for unranked ordered trees, called CF-HA, where word context-free languages are used instead of regular ones at the horizontal level. We show (Theorem 2) that CF-HA are preserved by rewrite closure using context-free rewrite rules. Context-free rewrite rules are the symmetric case of inverse context-free rules, i.e. rules with left-handside of the form $f(x)$. Some additional restrictions are assumed for this result, they cannot be relaxed as shown by the counter examples in Proposition 7-10.

Related works. Whether the rewrite closure of regular ranked trees languages is regular too is a problem that has been addressed in $[19,7,9,15,21,20,6]$. An important breakthrough of the proof in [15] (against former results) is that it works for TRS which are not left-linear. H. Ohsaki introduces equational tree automata for associative and commutative theories in [16] and study their closure properties for Boolean operations. T. Touili has studied the regular model checking problem for HA [22]. She shows how to compute the image of a HA language in one step of rewriting by a right-linear rewrite system. She also gives a procedure to compute an over-approximation of the rewrite closure of a HA. 
We rather compute exactly this closure for a class of non-linear rewrite systems. Our first main result (Theorem 1) can be viewed as a non trivial generalization of both [15] and [22], with proof techniques extending both former constructions.

C. Löding and A. Spelten [11] compute exact rewrite closure of HA for extensions of ground term rewriting and prefix word rewriting. These results cannot be compared to ours since in our case variables (that can be substituted by arbitrarily large hedges) allow non local hedge transformations.

There exists other rewriting notions like the top-down XML transformations [12] or the relabeling transducers of [18] but they do not cover our notion since either they use specific hedge traversal strategies or they are structurepreserving.

Layout of the paper. In Section 2 we introduce terms, hedges and the related rewriting concepts. In particular we define hedge rewriting systems (HRS) and context-free rewrite rules. In Section 3 we recall the hedge-automata classes HA and CF-HA that we shall investigate. In Section 4 we show that the class of HA languages, (i.e. recognized by HA) is preserved by rewrite closure for rewriting systems containing rules that are inverse context-free. In Section 5 we show that a class of context-free hedge rewrite systems preserves CF-HA languages. In both Sections 4 and 5, we also exhibit some counter-examples obtained when trying to relax the conditions on rules.

\section{Hedge Rewriting}

We consider a finite alphabet $\Sigma$ and an infinite set of variables $\mathcal{X}$. The set of terms over $\Sigma$ and $\mathcal{X}$ is $\mathcal{T}(\Sigma, \mathcal{X}):=\mathcal{X} \cup\{f(h) \mid f \in \Sigma, h \in \mathcal{H}(\Sigma, \mathcal{X})\}$ and the set $\mathcal{H}(\Sigma, \mathcal{X})$ of hedges over $\Sigma$ and $\mathcal{X}$ is the set of finite (possibly empty) sequences of terms of $\mathcal{T}(\Sigma, \mathcal{X})$. When $h$ is empty, $f()$ will be simply written $f$. We will sometimes consider a term as a hedge of length one, i.e. consider that $\mathcal{T}(\Sigma, \mathcal{X}) \subset \mathcal{H}(\Sigma, \mathcal{X})$. The sets of ground terms (terms without variables) and ground hedges are respectively denoted $\mathcal{T}(\Sigma)$ and $\mathcal{H}(\Sigma)$. A hedge $h \in \mathcal{H}(\Sigma, \mathcal{X})$ is called linear if every variable of $\mathcal{X}$ occurs at most once in $h$.

The set of variables occurring in a term $t \in \mathcal{T}(\Sigma, \mathcal{X})$ is denoted $\operatorname{var}(t)$. A substitution $\sigma$ is a mapping from $\mathcal{X}$ to $\mathcal{H}(\Sigma, \mathcal{X})$ of finite domain. The application of a substitution $\sigma$ to a hedge $h \in \mathcal{H}(\Sigma, \mathcal{X})$, denoted $h \sigma$, is the homomorphic extension of $\sigma$ to $\mathcal{H}(\Sigma, \mathcal{X})$, defined, for $t_{1}, \ldots, t_{n} \in \mathcal{T}(\Sigma, \mathcal{X})$, with $n \geq 0$, by $\left(t_{1} \ldots t_{n}\right) \sigma:=t_{1} \sigma \ldots t_{n} \sigma$ and $f(h) \sigma:=f(h \sigma)$.

The set of positions $\mathcal{P} o s(t)$ of a term $t \in \mathcal{T}(\Sigma, \mathcal{X})$ is a set of sequences of positive integers. The empty sequence, denoted $\varepsilon$, is the root position of a term. The subterm of $t$ at position $p$, denoted $\left.t\right|_{p}$, is defined by $\left.f\left(t_{1} \ldots t_{n}\right)\right|_{i p}:=\left.t_{i}\right|_{p}$ if $i \leq n$ and, $\left.f(h)\right|_{\varepsilon}:=f(h)$. The replacement in $t \in \mathcal{T}(\Sigma, \mathcal{X})$ of the subterm at position $p$ by $t^{\prime} \in \mathcal{T}(\Sigma, \mathcal{X})$ is denoted $t\left[t^{\prime}\right]_{p}$. The depth of a term is the maximal length of one of its positions.

A context is a linear hedge of $\mathcal{H}(\Sigma,\{x\})$, denoted $C[x]$. The application of a context $C[x]$ to a hedge $h$ is defined by $C[h]:=C\{x \mapsto h\}$. 
A hedge rewriting system (HRS) is a set of rewrite rules of the form $\ell \rightarrow r$ where $\ell \in \mathcal{T}(\Sigma, \mathcal{X}) \backslash \mathcal{X}$ and $r \in \mathcal{T}(\Sigma, \mathcal{X})(\ell$ and $r$ are respectively called $l h s$ and rhs of the rule). The rewrite relation $\overrightarrow{\mathcal{R}}$ of an HRS $\mathcal{R}$ is the binary relation on $\mathcal{H}(\Sigma, \mathcal{X})$ defined by $h \underset{\mathcal{R}}{\longrightarrow} h^{\prime}$ iff $h=\left(t_{1} \ldots t_{n}\right)$, there exists $i \leq n$, a position $p \in \mathcal{P}$ os $\left(t_{i}\right)$, a rule $\ell \rightarrow r \in \mathcal{R}$ and a substitution $\sigma$ such that $\left.t_{i}\right|_{p}=\ell \sigma$ and $h^{\prime}=t_{1} \ldots t_{i-1} t_{i}[r \sigma] t_{i+1} \ldots t_{n}$. The reflexive and transitive closure of $\overrightarrow{\mathcal{R}}$ is denoted $\underset{\mathcal{R}}{\stackrel{*}{\mathcal{L}} \text {. }}$.

Example 1. With $\mathcal{R}=\{g(x) \rightarrow x\}, \overrightarrow{\mathcal{R}}$ associates to a term $g(h)$ the hedge $h$ of its arguments. With $\mathcal{R}=\{g(x) \rightarrow g(a x b)\}, g(c) \stackrel{*}{\mathcal{R}} g\left(a^{n} c b^{n}\right)$ for every $n \geq 0$.

Given a set of terms $L \subseteq \mathcal{T}(\Sigma)$ and an HRS $\mathcal{R}$, we note $\mathcal{R}^{*}(L)$ the set $\{t \in$ $\mathcal{T}(\Sigma) \mid \exists s \in L, s \stackrel{*}{\mathcal{R}} t\}$. We restrict to terms (instead of hedges) because we are mainly interested in term languages below.

A rewrite rule $\ell \rightarrow r$ is called left-linear (resp. right-linear, linear) if $\ell$ (resp. $r$, both) is linear, left-ground (resp. right-ground) if $\ell \in \mathcal{T}(\Sigma)$ (resp. $r \in \mathcal{T}(\Sigma)$ ), collapsing if $r \in \operatorname{var}(\ell)$, it is called context-free if $\ell=f(x)$ with $x \in \mathcal{X}$ (it is not required that $x \in \operatorname{var}(r)$ however) and inverse context-free if $r \rightarrow \ell$ is context-free, prefix (resp. postfix) if $r=g\left(t_{0} \ldots t_{n} x\right)\left(\right.$ resp. $\left.r=g\left(x t_{0} \ldots t_{n}\right)\right)$ with $x \in \operatorname{var}(\ell)$ and no variable of $\ell$ occurs in the terms $t_{0}, \ldots, t_{n}$. A rewrite system is said to have one of the above properties if all its rules have this property.

Example 2. We give a few applications of our rewrite rules in the vein of [22]. A context-free rule $\operatorname{doc}(x) \rightarrow \operatorname{doc}(\mathrm{a} x \overline{\mathrm{a}})$ can be employed to introduce tags in an XML document. An inverse context-free rule can be used to eliminate comments $\operatorname{doc}(x$ comment $y \overline{\text { comment }}) \rightarrow \operatorname{doc}(x)$. Non left-linear inverse context-free rules are quite useful for processing list of items as in: $\operatorname{doc}($ todo $x \overline{\text { todo }} y$ done $x \overline{\operatorname{done}}) \rightarrow \operatorname{doc}(y)$.

Note that hedge rewriting cannot be reduced to term rewriting through encoding of unranked trees into ranked trees like the First-Child/Next-Sibling encoding, or the encoding used in stepwise automata. Consider for instance the hedge rewrite rule $f(a x a) \rightarrow f(x)$. For every $n \geq 0, f\left(a b^{n} a\right)$ reduces in one step to $f\left(b^{n}\right)$, however there is no finite term rewrite system that can simulate such reductions in one step. Given a term $t$ let us define inductively: $C_{t}^{0}=t$ and $C_{t}^{n}=b\left(\perp, C_{t}^{n-1}\right)$. Then $f\left(a b^{n} a\right)$ (resp. $\left.f\left(b^{n}\right)\right)$ is encoded by First-Child/NextSibling to $f\left(a\left(\perp, C_{a}^{n}\right), \perp\right)$ (resp. $f\left(C_{\perp}^{n}, \perp\right)$ ). Applying a term rewrite system to $f\left(a\left(\perp, C_{a}^{n}\right), \perp\right)$, for $n$ large enough, an instance of a variable of a left-hand side of an applicable rule should contain some subterm $C_{a}^{m}$, with $m>0$. This variable should also occur in the right-hand side to preserve the balance of $b$ symbols. But then $a$ should occur in the right-hand side too, contradiction.

\section{Hedge-Automata, Context-Free Hedge-Automata}

We recall now the definition of hedge-automata [13] (denoted HA) and the less known class of context-free hedge automata (denoted CF-HA) introduced in [17] 
and where they are shown to recognize the closure of regular (ranked) tree languages modulo associativity.

A hedge automaton (resp. context-free hedge automaton) is a tuple $\mathcal{A}=$ $\left(Q, \Sigma, Q^{\mathrm{f}}, \Delta\right)$ where $Q$ is a finite set of states, $\Sigma$ is an unranked alphabet, $Q^{\mathrm{f}} \subseteq Q$ is a set of final states, and $\Delta$ is a set of transitions of the form $f(L) \rightarrow q$ where $f \in \Sigma, q \in Q$ and $L \subseteq Q^{*}$ is a regular word language (resp. a context-free word language). When $\Sigma$ is clear from the context it is omitted in the tuple specifying $\mathcal{A}$.

We define the move relation between ground hedges in $\mathcal{T}(\Sigma \cup Q)$ as follows: for every terms $t, t^{\prime}$ we have $t \rightarrow \overrightarrow{\mathcal{A}} t^{\prime}$ if there exists a context $C[x]$ and a transition $f(L) \rightarrow q$ in $\Delta$ such that $t=C\left[f\left(q_{1} \ldots q_{n}\right)\right], q_{1} \ldots q_{n} \in L$ and $t^{\prime}=C[q]$. The relation $\underset{\mathcal{A}}{\mathcal{A}}$ is the transitive closure of $\overrightarrow{\mathcal{A}}$. Following [22], we extend $\overrightarrow{\mathcal{A}}$ to terms of $\mathcal{T}\left(\Sigma \cup 2^{Q^{*}}\right)$ as follows: $C\left[f\left(L_{1} \ldots L_{n}\right)\right] \underset{\mathcal{A}}{\longrightarrow} C[q]$ if there exists a rule $f(L) \rightarrow q$ in $\mathcal{A}$ such that $L_{1} \ldots L_{n} \subseteq L$ (in this definition, a lone state $q$ is considered as a singleton set $\{q\}$ ).

The language denoted by $L(\mathcal{A}, q)$ is the set of ground terms $t \in \mathcal{T}(\Sigma)$ such that $t \stackrel{*}{\mathcal{A}} q$. A term is accepted by $\mathcal{A}$ if there is $q \in Q^{\mathrm{f}}$ such that $t \in L(\mathcal{A}, q)$. The language denoted by $L(\mathcal{A})$ is the set of terms accepted by $\mathcal{A}$.

It is know that for both classes of automata $[13,17]$ membership and emptiness problems are decidable. Moreover HA are closed under Boolean operations.

We call a HA or CF-HA $\mathcal{A}=\left(Q, Q^{\mathrm{f}}, \Delta\right)$ normalized if for every $f \in \Sigma$ and every $q \in Q$, there is at most one transition rule $f\left(L_{f, q}\right) \rightarrow q$ in $\Delta$. Every HA (resp. CF-HA) can be transformed into a normalized HA (resp. CF-HA) in polynomial time by replacing every two rules $f\left(L_{1}\right) \rightarrow q$ and $f\left(L_{2}\right) \rightarrow q$ by $f\left(L_{1} \cup L_{2}\right) \rightarrow q$.

A HA $\mathcal{A}=\left(Q, Q^{\mathrm{f}}, \Delta\right)$ is called deterministic iff for all two transitions rules $f\left(L_{1}\right) \rightarrow q_{1}$ and $f\left(L_{2}\right) \rightarrow q_{2}$ in $\Delta$, either $L_{1} \cap L_{2}=\emptyset$ or $q_{1}=q_{2}$. It is called complete if for all $f \in \Sigma$ and and $w \in Q^{*}$, there exists at least one rule $f(L) \rightarrow$ $q \in \Delta$ such that $w \in L$. When $\mathcal{A}$ is deterministic (resp. complete), for all $t \in$ $\mathcal{T}(\Sigma)$, there exists at most (resp. at least) one state $q \in Q$ such that $t \in L(\mathcal{A}, q)$.

Every HA can be completed by adding a sink state (and using the closure properties of regular languages). A determinization procedure (with a subset construction) which preserves completeness is described in Section 4.1 (see also [4]).

\subsection{Epsilon- and Collapsing Transitions}

We can extend HA and CF-HA with $\varepsilon$-transitions of the form $q \rightarrow q^{\prime}$, where $q$ and $q^{\prime}$ are states, without augmenting the respective expressiveness of these classes. We also consider the extensions of HA (resp. CF-HA), with collapsing transitions of the form $L \rightarrow q$ where $L$ is a regular (resp. CF) language and $q$ is a state. The move relation for the extended set of transitions is defined as for $\mathrm{HA}$ and CF-HA for standard transition and by $C\left[q_{1} \ldots q_{k}\right] \underset{\mathcal{A}}{\longrightarrow} C[q]$ if $L \rightarrow q$ is a collapsing transition of $\mathcal{A}$ and $q_{1} \ldots q_{k} \in L$. Note that the collapsing transition $L \rightarrow q$ is never applied at the root position (i.e. the above context $C$ cannot be a variable) because $\mathrm{HA}$ and $\mathrm{CF}-\mathrm{HA}$ are limited to the recognition of terms only (and not hedges). 
Unlike $\varepsilon$-transitions, collapsing transitions strictly extend HA in expressiveness. However, we show that they can be eliminated for CF-HA.

Proposition 1. For every extended $H A$ or $C F-H A$ with collapsing transitions $\mathcal{A}$, there exists a $C F-H A \mathcal{A}^{\prime}$ (without collapsing transitions) such that $L\left(\mathcal{A}^{\prime}\right)=$ $L(\mathcal{A})$.

Proof. Assume that $L \rightarrow q$ is a collapsing transition of $\mathcal{A}$. Then we get a CF-HA $\mathcal{A}^{\prime}$ such that $L\left(\mathcal{A}^{\prime}\right)=L(\mathcal{A})$ by replacing every transition $f\left(L_{1}\right) \rightarrow q_{1}$ by the transition $f\left(L_{2}\right) \rightarrow q_{1}$ where $L_{2}$ is the context-free word language generated by the grammar $G_{2}$ as follows. We consider a context-free grammar $G$ for $L$ (resp. $G_{1}$ for $L_{1}$ ) with axiom $X$ (resp. $X_{1}$ ). The axiom of $G_{2}$ is $X_{1}$ and the set of productions in $G_{2}$ contains $\left.i\right) G\left[q \leftarrow X_{q}\right] \cup G_{1}\left[q \leftarrow X_{q}\right]$ i.e. the terminal $q$ is replaced by a non terminal $X_{q}$ and $i i$ ) we add to these rules the production: $X_{q}:=$ $q \mid X$. We can iterate this construction to eliminate all collapsing transitions.

Proposition 2. There exists an extended HA with collapsing transitions whose language is not a $H A$ language.

Proof. Consider the extended HA $\mathcal{A}=\left(\left\{q, q_{a}, q_{b}, q_{\mathrm{f}}\right\},\{g, a, b, c\},\left\{q_{\mathrm{f}}\right\}, \Delta\right)$ where

$$
\Delta=\left\{c \rightarrow q, a \rightarrow q_{a}, b \rightarrow q_{b}, g(q) \rightarrow q_{\mathrm{f}}, q_{a} q q_{b} \rightarrow q\right\}
$$

Its recognized language is $\left\{g\left(a^{n} c b^{n}\right) \mid n \geq 0\right\}$ and this is not a HA language.

\subsection{Decision Problems}

The problem of ground reachability and ground joinability are to decide that, given two ground terms $s, t \in \mathcal{T}(\Sigma)$ and a HRS $\mathcal{R}$, whether, $s \stackrel{*}{\mathcal{R}} t$, respectively, $s \stackrel{*}{\mathcal{R}} \circ \stackrel{*}{\mathcal{R}} t$.

Regular hedge model checking is the problem to decide, given two HA languages $L_{\text {init }}$ and $L_{\text {err }}$ and a $\operatorname{HRS} \mathcal{R}$ whether $\mathcal{R}^{*}\left(L_{\text {init }}\right)$ contains a term of $L_{\text {err }}$. Ground reachability is reducible to regular hedge model-checking. Indeed, given $s, t$ and $\mathcal{R}, s \underset{\mathcal{R}}{\stackrel{*}{\longrightarrow}} t$ iff $\mathcal{R}^{*}(\{s\}) \cap\{t\} \neq \emptyset$. Note also that if ground-reachability (hence regular hedge model-checking) is undecidable for a class of HRS, then $\mathcal{R}^{*}(L)$ is not recursive in general when $\mathcal{R}$ is in this class and $L$ is a HA or CF-HA. Indeed, by definition $s \stackrel{*}{\mathcal{R}} t$ iff $t \in \mathcal{R}^{*}(\{s\})$.

\section{Closure of Regular Hedge Automata Languages}

In this section, we prove one result of preservation of HA language for a class of HRS, and give several counter example showing that the restrictions defining this class of HRS are necessary. 


\subsection{Inverse Context-Free Rewrite Rules}

Theorem 1. The closure $\mathcal{R}^{*}(L)$ of a HA language $L \subseteq \mathcal{T}(\Sigma)$ under rewriting by an inverse context-free $H R S \mathcal{R}$ is a HA language.

Proof. Let $\mathcal{A}=\left(Q, Q^{\mathrm{f}}, \Delta\right)$ be a complete and normalized HA recognizing $L$. We shall construct below a finite sequence of HA $\left(\mathcal{A}_{i}\right)_{0<i<h}$ whose last element recognizes $\mathcal{R}^{*}(L)$. Our construction uses elements of [15] and [22], but it is not a simple combination of both. Indeed, on one side we generalize [22] to an unbounded number of rewriting steps, and on the other side we generalize [15] to unranked tree languages. Both generalizations are non-trivial and require new constructions and new conditions.

For each $f \in \Sigma, q \in Q$, we note $L_{f, q}$ the language in the transition (assumed unique) $f\left(L_{f, q}\right) \rightarrow q \in \Delta$. We construct first from $\mathcal{A}$ a deterministic, complete and normalized HA $\mathcal{A}_{\mathrm{d}}=\left(Q_{\mathrm{d}}, Q_{\mathrm{d}}^{\mathrm{f}}, \Delta_{\mathrm{d}}\right)$ recognizing $L$. The HA $\mathcal{A}_{\mathrm{d}}$ is obtained by a subset construction, see e.g. [4], with $Q_{\mathrm{d}}:=2^{Q}, Q_{\mathrm{d}}^{\mathrm{f}}:=\left\{s \in Q_{\mathrm{d}} \mid s \cap Q^{\mathrm{f}} \neq \emptyset\right\}$ and $\Delta_{\mathrm{d}}:=\left\{f\left(L_{f, s}\right) \rightarrow s \mid f \in \Sigma, s \subseteq Q\right\}$ where $L_{f, s}:=\left(\bigcap_{q \in s} S_{f, q}\right) \backslash\left(\bigcup_{q \notin s} S_{f, q}\right)$ and $S_{f, q}=\left\{s_{1} \ldots s_{n} \in Q_{\mathrm{d}}^{*} \mid \exists q_{1} \in s_{1}, \ldots, q_{n} \in s_{n}, q_{1} \ldots q_{n} \in L_{f, q}\right\}^{3}$.

Next, following the approach of [22], we define first the set of languages of $Q_{\mathrm{d}}^{*}$ that will be used in the transitions of the $\mathcal{A}_{i}$ 's constructed below. However, we must consider here a bigger set than [22] in order to deal with non linear variables in lhs of rules. Let $\mathcal{L}$ be the smallest set of subsets of $Q_{\mathrm{d}}^{*}$ such that

$i$. all $L_{f, s}$ (for $f \in \Sigma$ and $s \in Q_{\mathrm{d}}$ ) and $Q_{\mathrm{d}}^{*}$ are in $\mathcal{L}$,

ii. if $L \in \mathcal{L}$ and $u, v \in Q_{\mathrm{d}}^{*}$, then $u^{-1} L v^{-1} \in \mathcal{L}$, where

$$
u^{-1} L v^{-1}:=\left\{w \in Q_{\mathrm{d}}^{*} \mid u w v \in L\right\},
$$

iii. if $L_{1}, L_{2} \in \mathcal{L}$ then $L_{1} \cap L_{2} \in \mathcal{L}$,

iv. if $L_{1}, L_{2} \in \mathcal{L}$ then $L_{1} \backslash L_{2} \in \mathcal{L}$.

Note that the condition $Q_{\mathrm{d}}^{*} \in \mathcal{L}$ in $i$ together with iii and $i v$ imply that $\mathcal{L}$ is also closed under union (if $L_{1}, L_{2} \in \mathcal{L}$ then $L_{1} \cup L_{2} \in \mathcal{L}$ ), by De Morgan's Law.

Let us show that $\mathcal{L}$ is finite and that all its members are regular languages. First, let us note that $\mathcal{L}_{1}$, the smallest set satisfying $i$ and $i i$ above, is a finite set of regular languages of $Q_{\mathrm{d}}^{*}$, since every $L_{f, q}$ is regular by hypothesis. The closure $\mathcal{L}_{2}$ of $\mathcal{L}_{1}$ under iii and then $i v$ is also a finite set of regular languages. The following lemma shows that $\mathcal{L}_{2}$ fulfills $i$, i.e. that $\mathcal{L}_{2}=\mathcal{L}$.

Lemma 1. For all $L_{1}, L_{2} \subseteq Q_{\mathrm{d}}^{*}, u_{1}, u_{2}, v_{1}, v_{2}, u, v \in Q^{*}$, we have $u^{-1}\left(u_{1}^{-1} L_{1} v_{1}^{-1} \cap u_{2}^{-1} L_{2} v_{2}^{-1}\right) v^{-1}=\left(u_{1} u\right)^{-1} L_{1}\left(v v_{1}\right)^{-1} \cap\left(u_{2} u\right)^{-1} L_{2}\left(v v_{2}\right)^{-1}$, $u^{-1}\left(u_{1}^{-1} L_{1} v_{1}^{-1} \backslash u_{2}^{-1} L_{2} v_{2}^{-1}\right) v^{-1}=\left(u_{1} u\right)^{-1} L_{1}\left(v v_{1}\right)^{-1} \backslash\left(u_{2} u\right)^{-1} L_{2}\left(v v_{2}\right)^{-1}$.

Proof. The set in the left-hand-side of the first identity in Lemma 1 is $A=$ $\left\{\ell \mid u \ell v \in\left\{\ell^{\prime} \mid u_{1} \ell^{\prime} v_{1} \in L_{1}\right.\right.$ and $\left.\left.u_{2} \ell^{\prime} v_{2} \in L_{2}\right\}\right\}$, and the set in its right hand side is $B=\left\{\ell \mid u_{1} u \ell v v_{1} \in L_{1}\right.$ and $\left.u_{2} u \ell v v_{2} \in L_{2}\right\}$. If $\ell \in A$, then $u_{1} u \ell v v_{1} \in L_{1}$

\footnotetext{
${ }^{3}$ Note that $S_{f, q}$ and $L_{f, s}$ are indeed regular languages, see [4].
} 
and $u_{2} u \ell v v_{2} \in L_{2}$, hence $\ell \in B$. Conversely, if $\ell \in B$, then $u \ell v \in u_{1}^{-1} L_{1} v_{1}^{-1} \cap$ $u_{2}^{-1} L_{2} v_{2}^{-1}$, hence $\ell \in A$. The proof is very similar for the identity with the complementation.

Let us now construct the HA $\mathcal{A}_{0}, \ldots, \mathcal{A}_{h}$ as announced. The set of states and final states of each of these HA are respectively $Q_{\mathrm{d}}$ and $Q_{\mathrm{d}}^{\mathrm{f}}$. We give below an iterative construction of the respective transition sets $\Delta_{i}, 0 \leq i \leq h$.

Let $\Delta_{0}=\Delta_{\mathrm{d}}$. Assume that $\Delta_{i}$ has been constructed and contains one transition $f\left(L_{f, s}^{i}\right) \rightarrow s$ for every $f \in \Sigma$ and $s \in Q_{\mathrm{d}} ; \Delta_{i+1}$ is obtained from $\Delta_{i}$ as follows: choose (non deterministically) an inverse context-free rewrite rule $\ell \rightarrow g(x) \in \mathcal{R}$, and a substitution $\tau: \operatorname{var}(\ell) \cup\{x\} \rightarrow\left\{L^{\prime} \in \mathcal{L} \mid \forall s_{1} \ldots s_{k} \in L^{\prime}, \forall j \leq k, L\left(\mathcal{A}_{i}, s_{j}\right) \neq\right.$ $\emptyset\}$, such that $\ell \tau \underset{\mathcal{A}_{i}}{\stackrel{*}{\longrightarrow}} s^{\prime} \in Q_{\mathrm{d}}$. Let $L^{\prime}=x \tau$ (note that if the variable $x$ does not occur in $\ell$, then $L^{\prime}$ is an arbitrary language of $\mathcal{L}$ of sequences of states reachable by $\left.\mathcal{A}_{i}\right) ; \Delta_{i+1}$ is obtained as follows: for each $s \in Q_{\mathrm{d}}$,

1. replace the rule $g\left(L_{g, s}^{i}\right) \rightarrow s$ by $g\left(L_{g, s}^{i} \cap L^{\prime}\right) \rightarrow s \cup s^{\prime}$ and $g\left(L_{g, s}^{i} \backslash L^{\prime}\right) \rightarrow s$

2. after this operation, normalize the set of transition rules with the operation described in Section 3 (page 5). (Note that if $s^{\prime} \subseteq s$ then the normalization merges the 2 rules and regenerate $g\left(L_{g, s}^{i}\right) \rightarrow s$.)

The idea behind this construction is that if $s^{\prime}$ is reachable from a lhs $\ell \tau$ of rewrite rule, then the states in $s^{\prime}$ must also be reachable from the corresponding rhs $g(x \tau)$. Note that for all transitions $g(L) \rightarrow s$ produced by the algorithm, we have $L \in \mathcal{L}$ (even after normalization), according to the closure properties of this set. Since $\mathcal{L}$ and the set of states $s$ is finite (no new state is added) this shows that the construction terminates say with a HA $\mathcal{A}_{h}$ that will be $\operatorname{denoted} \mathcal{A}^{*}$.

We can also show the following invariant: every $\mathcal{A}_{i}$ constructed in the algorithm is deterministic, complete and normalized. Indeed, assume that $\mathcal{A}_{i}$ has these properties. If $s^{\prime} \subseteq s$ no transition is added and the invariant is trivially preserved; hence we can assume now $s^{\prime} \not \subset s$. If another rule $g\left(L_{g, s \cup s^{\prime}}^{i}\right) \rightarrow s \cup s^{\prime}$ was in $\Delta_{i}$ it is merged with $g\left(L_{g, s}^{i} \cap L^{\prime}\right) \rightarrow s \cup s^{\prime}$ by normalization producing the rule $g\left(\left(L_{g, s}^{i} \cap L^{\prime}\right) \cup L_{g, s \cup s^{\prime}}^{i}\right) \rightarrow s \cup s^{\prime}$. Hence there is at most one $L_{g, s \cup s^{\prime}}^{i+1}=\left(L_{g, s}^{i} \cap L^{\prime}\right) \cup L_{g, s \cup s^{\prime}}^{i}$ such that $g\left(L_{g, s \cup s^{\prime}}^{i+1}\right) \rightarrow s \cup s^{\prime} \in \Delta_{i+1}$. Note also that there is at most one $L_{g, s}^{i+1}=L_{g, s}^{i} \backslash L^{\prime}$ such that $g\left(L_{g, s}^{i+1}\right) \rightarrow s \in \Delta_{i+1}$. It is easy to see (from the fact that $\mathcal{A}_{i}$ is deterministic and normalized) that $L_{g, s \cup s^{\prime}}^{i+1}, L_{g, s}^{i+1}$, and $L_{g, s^{\prime \prime}}^{i+1}$, for all $s^{\prime \prime} \notin\left\{s, s^{\prime}\right\}$, are pairwise disjoint, hence $\mathcal{A}_{i+1}$ is deterministic. From the facts that $\mathcal{A}_{i}$ is complete and that $L_{g, s}^{i} \cap L^{\prime}$ and $L_{g, s}^{i} \backslash L^{\prime}$ form a partition of $L_{g, s}^{i}$, we deduce that $\mathcal{A}_{i+1}$ is also complete.

We show now that $L\left(\mathcal{A}^{*}\right)=\mathcal{R}^{*}(L)$. The proof of the direction $L\left(\mathcal{A}^{*}\right) \subseteq \mathcal{R}^{*}(L)$ relies on the following lifting lemma.

Lemma 2. For all $i \geq 0, t \in \mathcal{T}(\Sigma, \mathcal{X}), \sigma: \operatorname{var}(t) \rightarrow \mathcal{H}(\Sigma)$ and $\theta: \operatorname{var}(t) \rightarrow Q_{\mathrm{d}}^{*}$ such that for all $x \in \operatorname{var}(t), x \sigma$ and $x \theta$ have the same length, if $t \theta \underset{\mathcal{A}_{i}}{\longrightarrow} s_{0} \in Q_{\mathrm{d}}$, and for all $x \in \operatorname{var}(t)$, all components $\left.(x \theta)\right|_{j}$ of $x \theta$ (state of $Q_{\mathrm{d}}$ ) and $\left.q \in(x \theta)\right|_{j}$, there exists $u \in L\left(\mathcal{A}_{i}, q\right)$ such that $\left.u \stackrel{*}{\mathcal{R}}(x \sigma)\right|_{j}$, then for all $q^{\prime} \in s_{0}$, there exists $v \in L\left(\mathcal{A}_{i}, q^{\prime}\right)$ s.t. $v \stackrel{*}{\mathcal{R}} t \sigma$. 
Proof. We make an induction on $i$.

Base case $(i=0)$. We have $\mathcal{A}_{0}=\mathcal{A}_{\mathrm{d}}$ and since $t \sigma \underset{\mathcal{A}_{\mathrm{d}}}{\stackrel{*}{\longrightarrow}} s_{0}$, by construction of $\mathcal{A}_{\mathrm{d}}$, for all $q_{0} \in s_{0}, t \sigma \stackrel{*}{\mathcal{A}} q_{0}$.

Induction step $(i+1)$. We assume that the property is true for $i$ and prove the property for $i+1$ by induction on the number $n^{\prime}$ of applications of a rule of $\Delta_{i+1} \backslash \Delta_{i}$ in the reduction $t \theta \underset{\mathcal{A}_{i+1}}{\stackrel{*}{\longrightarrow}} s_{0}$.

Base case $\left(n^{\prime}=0\right)$. If there are no rules of $\Delta_{i+1} \backslash \Delta_{i}$ in the above reduction, then $t \theta \underset{\mathcal{A}_{i}}{\stackrel{*}{\longrightarrow}} s_{0}$ and we apply the induction hypothesis (on $i$ ).

Induction step $\left(n^{\prime}+1\right)$. Let $\rho$ be a rule in $\Delta_{i+1} \backslash \Delta_{i}$ applied at the position $p$ of $t \theta$ in the above reduction sequence. Let $z \notin \operatorname{var}(t)$ be a fresh variable and let $\sigma^{\prime}=\left\{\left.z \mapsto t \sigma\right|_{p}\right\}$. With this definition, $t \sigma=t[z]_{p} \sigma \cup \sigma^{\prime}$. We have the reduction $t \sigma \underset{A_{i+1}}{\stackrel{*}{\longrightarrow}} s_{0}$ described in the top line of Figure 1 .

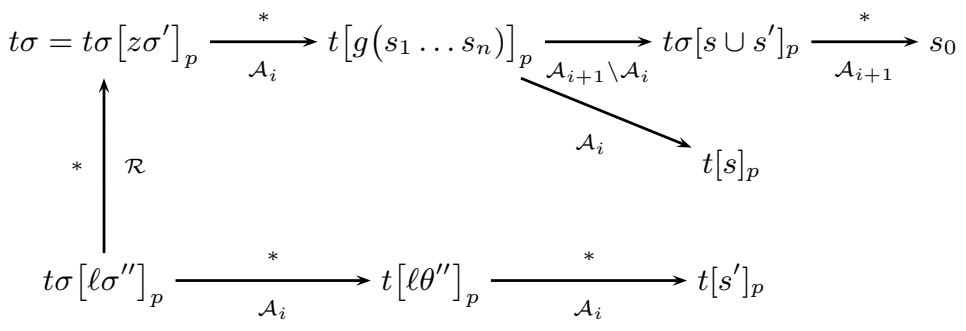

Fig. 1. Proof of Lemma $2, L\left(\mathcal{A}^{*}\right) \subseteq \mathcal{R}^{*}(L)$.

Assume that $\rho=g\left(\left(L_{g, s}^{i} \cap L^{\prime}\right) \cup L_{g, s \cup s^{\prime}}^{i}\right) \rightarrow s \cup s^{\prime}$ and that it has been added using an inverse context-free rewrite rule $\ell \rightarrow g(z) \in \mathcal{R}$. Let $\tau: \operatorname{var}(\ell) \rightarrow \mathcal{L}$ be the substitution used for the construction of the above rule, such that $\ell \tau \stackrel{*}{\mathcal{A}_{i}}$ $s^{\prime} \in Q_{\mathrm{d}}$.

By hypothesis on $\tau$, to each $y \in \operatorname{var}(\ell)$ we can associate a hedge $h$ which is reduced by $\mathcal{A}_{i}$ to a sequence of states of $Q_{\mathrm{d}}$ in the language of $y \tau$. This permits to define two substitutions $\sigma^{\prime \prime}: \operatorname{var}(\ell) \rightarrow \mathcal{H}(\Sigma)$ and $\theta^{\prime \prime}: \operatorname{var}(\ell) \ni y \mapsto \bar{s} \in y \tau$, making possible the reduction (with $\mathcal{A}_{i}$ ) at the bottom line of Figure 1 and such that moreover $t\left[\ell \sigma^{\prime \prime}\right]_{p} \underset{\mathcal{R}}{\longrightarrow} t \sigma$. The latter reduction is obtained by letting $z \sigma^{\prime \prime}:=\left.z \sigma^{\prime}\right|_{1}$.

By induction hypothesis, (on the number of applications of a rule of $\Delta_{i+1} \backslash \Delta_{i}$ in $\ell \theta^{\prime \prime} \underset{\mathcal{A}_{i}}{\stackrel{*}{*}} s^{\prime}$ ), for all $q^{\prime} \in s^{\prime}$, there exists $v \in L\left(\mathcal{A}, q^{\prime}\right)$ such that $v \underset{\mathcal{R}}{\stackrel{*}{\longrightarrow}} \ell \sigma^{\prime \prime}$. Moreover, by hypothesis on the rule $\rho$, the sequence of states $s_{1} \ldots s_{n}$ at position $p 1$ in the reduction $t \sigma \underset{\mathcal{A}_{i+1}}{\stackrel{*}{\longrightarrow}} s_{0}$ (see Figure 1) belongs to $L_{g, s}^{i}$. Hence we have a reduction $z \sigma^{\prime} \underset{\mathcal{A}_{i}}{\stackrel{*}{\longrightarrow}} s$ and by induction hypothesis, for all $q \in s$, there exists $u \in L(\mathcal{A}, q)$ such that $u \stackrel{*}{\mathcal{R}} z \sigma^{\prime}$. Altogether, for all $q \in s \cup s^{\prime}$, there exists 
$u \in L(\mathcal{A}, q)$ such that $u \stackrel{*}{\stackrel{\mathcal{R}}{\longrightarrow}} z \sigma^{\prime}$. Letting $\theta^{\prime}=\left\{z \mapsto s \cup s^{\prime}\right\}$, we can apply the induction hypothesis to $t \sigma\left[z \theta^{\prime}\right]_{p}$, because there is at least one application of $\Delta_{i+1} \backslash \Delta_{i}$ less in $t \sigma\left[z \theta^{\prime}\right]_{p} \underset{\mathcal{A}_{i+1}}{\stackrel{*}{\longrightarrow}} s_{0}$ than in $t \theta_{p} \underset{\mathcal{A}_{i+1}}{\stackrel{*}{\longrightarrow}} s_{0}$. Hence, for all $q \in s_{0}^{\prime}$, there exists $u \in L(\mathcal{A}, q)$ such that $u \stackrel{*}{\mathcal{R}} t \sigma$.

The case where a rule $\rho=g\left(L_{f, s}^{i} \backslash L^{\prime}\right) \rightarrow s$ is added is simpler, because, in order to apply the induction hypothesis, we only need to show that for all $q \in s$, there exists $u \in L(\mathcal{A}, q)$ such that $u \stackrel{*}{\mathcal{R}} z \sigma^{\prime}$ in the first case This can be done as before.

$$
\text { (end of the proof of Lemma 2) }
$$

Now, for the particular case of Lemma 2 where $t \in \mathcal{T}(\Sigma)$, we have that if $t \underset{\mathcal{A}_{i}}{\stackrel{*}{\longrightarrow}} s_{0}$, for some $i$ and $s_{0} \in Q_{\mathrm{d}}^{\mathrm{f}}$, for all $q^{\mathrm{f}} \in s_{0}$, where $q^{\mathrm{f}}$ is a final state of $\mathcal{A}$, there exists $u \in L\left(\mathcal{A}, q^{\mathrm{f}}\right) \subseteq L(\mathcal{A})$ such that $u \underset{\mathcal{R}}{\stackrel{*}{\mathcal{R}}} t$. This terminates the proof of the direction $L\left(\mathcal{A}^{*}\right) \subseteq \mathcal{R}^{*}(L)$.

For the direction $L\left(\mathcal{A}^{*}\right) \supseteq \mathcal{R}^{*}(L)$, assume that $t \in L(\mathcal{A})$ and that $t \stackrel{*}{\mathcal{R}} t^{\prime}$. We show by induction on the length of this reduction sequence that $t^{\prime} \in L\left(\mathcal{A}_{i}\right)$ for some $i$.

Base case $\left(t=t^{\prime}\right)$. It is immediate by construction of $\mathcal{A}_{0}$.

Induction step. Assume that the last step in $t \underset{\mathcal{R}}{\stackrel{*}{\longrightarrow}} t^{\prime}$ involves an inverse contextfree rule $\ell \rightarrow g(x) \in \mathcal{R}$ at a position $p$ and with a ground substitution $\sigma$ : $\operatorname{var}(\ell) \rightarrow \mathcal{H}(\Sigma)$, as described in the the top line of the diagram in Figure 2.

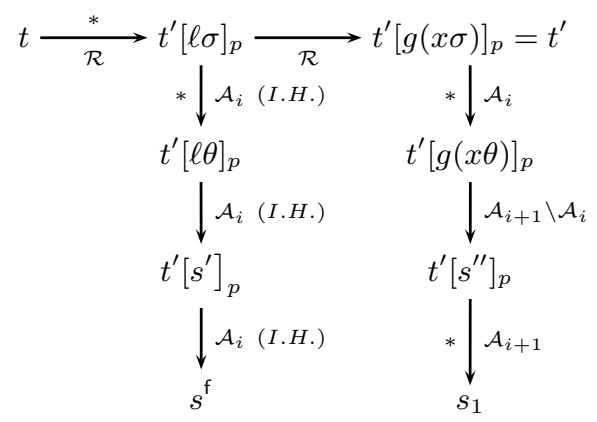

Fig. 2. Proof of $\mathcal{R}^{*}(L) \subseteq L\left(\mathcal{A}^{*}\right)$.

By induction hypothesis, $t^{\prime}[\ell \sigma]_{p} \in L\left(\mathcal{A}_{i}\right)$ for some $i$. Let us consider the reduction of this term to a final state $s^{\mathrm{f}}$ of $\mathcal{A}_{i}$ depicted in the second column of the diagram in Figure 2. Since $\mathcal{A}_{i}$ is deterministic, there exists a substitution $\theta: \operatorname{var}(\ell) \rightarrow Q_{\mathrm{d}}^{*}$ such that $t^{\prime}[\ell \sigma]_{p} \underset{\mathcal{A}_{i}}{\stackrel{*}{\longrightarrow}} t^{\prime}[\ell \theta]_{p}$ and $t^{\prime}[\ell \theta]_{p} \underset{\mathcal{A}_{i}}{\longrightarrow} t^{\prime}\left[s^{\prime}\right]_{p} \underset{\mathcal{A}_{i}}{\longrightarrow} s^{\mathrm{f}}$. Since $\mathcal{A}_{i}$ is complete, there exists a substitution $\tau: \operatorname{var}(\ell) \rightarrow\left\{L^{\prime} \in \mathcal{L} \mid \forall s_{1} \ldots s_{k} \in\right.$ $\left.L^{\prime}, \forall j \leq k, L\left(\mathcal{A}_{i}, s_{j}\right) \neq \emptyset\right\}$, such that for all $x \in \operatorname{var}(\ell), x \theta \in x \tau$. By definition of the relation $\underset{\mathcal{A}_{i}}{\stackrel{*}{\longrightarrow}}$ extended to $\mathcal{T}(\Sigma, \mathcal{L}), \ell \tau \underset{\mathcal{A}_{i}}{\stackrel{*}{\longrightarrow}} s^{\prime}$. 
Hence this $\tau$ is as in of the construction. It follows that a rule is added which permits the reduction $t^{\prime}[g(x \theta)]_{p} \underset{\mathcal{A}_{i+1}}{\stackrel{*}{\longrightarrow}} s^{\prime \prime}$ where $s^{\prime \prime}=s \cup s^{\prime}$ (the case $s^{\prime \prime}=s$ is not possible because $x \theta \in x \tau)$. We have a reduction from $t^{\prime}$ to a final state thank to the following technical lemma which state the monotonicity of the relation $\underset{\mathcal{A}_{i}}{\stackrel{*}{\longrightarrow}}$ wrt state inclusion and context application.

Lemma 3. For all $i \geq 0$, all context $C \in \mathcal{T}(\Sigma,\{x\})$ and $s_{0}, s_{0}^{\prime} \in Q_{\mathrm{d}}$ such that $s_{0} \subseteq s_{0}^{\prime}$ if $C\left[s_{0}\right] \underset{\mathcal{A}_{i}}{\stackrel{*}{\longrightarrow}} s \in Q_{\mathrm{d}}$, then $C\left[s_{0}^{\prime}\right] \underset{\mathcal{A}_{i}}{\stackrel{*}{\longrightarrow}} s^{\prime} \supseteq s$.

Proof. The proof is an induction on the structure of $C$.

Base case $(C=x)$. In this case, the result is immediate by hypothesis.

Induction step $\left(C=f\left(C_{1} \ldots C_{n}\right)\right)$. We have $C\left[s_{0}\right] \underset{\mathcal{A}_{i}}{\stackrel{*}{\longrightarrow}} f\left(s_{1} \ldots s_{n}\right) \underset{\mathcal{A}_{i}}{\longrightarrow} s$. By induction hypothesis, for all $j \leq n, C_{j}\left[s_{0}^{\prime}\right] \underset{\mathcal{A}_{0}}{\stackrel{*}{\longrightarrow}} s_{j}^{\prime} \supseteq s_{j}$. Since $\mathcal{A}_{i}$ is deterministic and complete, there exists a unique $s^{\prime}$ such that $f\left(s_{1}^{\prime} \ldots s_{n}^{\prime}\right) \underset{\mathcal{A}_{i}}{\longrightarrow} s^{\prime}$. We show that $s^{\prime} \subseteq s$ by induction on $i$.

Base case $(i=0)$. This case follows from the construction of $\mathcal{A}_{0}$, with the determinization procedure.

Induction step $(i=k+1)$. If $f\left(s_{1}^{\prime} \ldots s_{n}^{\prime}\right) \underset{\Delta_{k}}{\longrightarrow} s^{\prime}$, then we can conclude by induction hypothesis. If $f\left(s_{1}^{\prime} \ldots s_{n}^{\prime}\right) \underset{\Delta_{k+1} \backslash \Delta_{k}}{\longrightarrow} s^{\prime}$, then let $s^{\prime \prime}$ be the state of $Q_{\mathrm{d}}$ such that $s_{1}^{\prime} \ldots s_{n}^{\prime} \in L_{f, s^{\prime \prime}}^{k}$. This state exists and is unique because $\mathcal{A}_{k}$ is deterministic and complete, and by induction hypothesis (on $k$ ), $s \subseteq s^{\prime \prime}$. An analyze of the cases which may have permit the construction the transition $f\left(L_{f, s^{\prime}}^{k+1}\right) \rightarrow s^{\prime} \in \Delta_{k+1} \backslash \Delta_{k}$ used above shows that this rule replaces $f\left(L_{f, s^{\prime \prime}}^{k}\right) \rightarrow$ $s^{\prime \prime}$ and that, $s^{\prime \prime} \subseteq s^{\prime}$. Hence $s \subseteq s^{\prime}$.

With Lemma 3, we have $t^{\prime} \underset{\mathcal{A}_{i+1}}{\stackrel{*}{\longrightarrow}} s_{1}$ with $s_{1} \supseteq s^{\mathrm{f}}$. Hence $t^{\prime} \in L\left(\mathcal{A}_{i+1}\right)$.

(end of the proof of Theorem 1)

Corollary 1. Ground reachability, ground joinability and regular hedge modelchecking are decidable for inverse context-free HRS.

We present in the next subsections (4.2-4.4) some counter examples showing that relaxing the assumption on $\mathcal{R}$ in Theorem 1 invalidate the result.

\subsection{Collapsing Rewrite Rules}

Collapsing rules preserve regularity of term languages [15] when the function symbols are ranked. Indeed, in this case, if $\mathcal{R}$ is left-linear and collapsing, a tree automaton (TA) recognizing $L$ can be completed into a TA recognizing $\mathcal{R}^{*}(L)$ just by the iterated addition of $\varepsilon$-transitions of the form $x \tau \rightarrow q$ when there is $\ell \rightarrow x \in \mathcal{R}$ and a substitution $\tau: \operatorname{var}(\ell) \rightarrow Q$ such that $\ell \tau \stackrel{*}{\mathcal{A}} q$. When $\mathcal{R}$ is just collapsing (not left-linear), the construction requires determinism and hence is more complicated but the idea is the same [15]. 
In the case of unranked terms and HA, if we want to follow the principles of the construction of Section 4.1, we need to add collapsing transitions and not just $\varepsilon$-transitions. But the addition of collapsing transitions does not preserve HA languages (Proposition 1). The following proposition shows that the above construction is actually not possible for collapsing rewrite rules.

Proposition 3. $\mathcal{R}^{*}(L)$ is not a HA language in general when $L$ is a HA language and $\mathcal{R}$ is a linear collapsing $H R S$.

Proof. We use the principle of the construction in the proof of Proposition 1. Let $\Sigma=\{f, g, a, b, c\}$, let $L$ be the language of the HA

$$
\mathcal{A}=\left(\left\{q, q_{a}, q_{b}, q_{\mathrm{f}}\right\},\left\{q_{\mathrm{f}}\right\},\left\{c \rightarrow q, a \rightarrow q_{a}, b \rightarrow q_{b}, g\left(q_{a} q q_{b}\right) \rightarrow q, f(q) \rightarrow q_{\mathrm{f}}\right\}\right)
$$

and let $\mathcal{R}=\{g(x) \rightarrow x\}$. Assume that $\mathcal{R}^{*}(L)$ is a HA language. Its intersection with the HA language $\left\{f\left(a^{*} c b^{*}\right)\right\}$ is $\left\{f\left(a^{n} c b^{n}\right) \mid n \geq 0\right\}$. It is not a HA language. This contradicts the fact that HA languages are closed under intersection.

Note that the completion of the above $\mathcal{A}$, following the procedure in the proof of Theorem 1, would add the collapsing transition $q_{a} q q_{b} \rightarrow q$.

\subsection{Flat Linear Rewrite Rules}

In the case of ranked terms, it is known [15] that regularity of tree languages is preserved under rewriting with systems with right-linear rules of the form $\ell \rightarrow f\left(u_{1}, \ldots, u_{n}\right)$ where $f$ has arity $n$ and each $u_{i}(i \leq n)$ is either a ground term or a variable of $\operatorname{var}(\ell)$. We call such a rule flat if its lhs and rhs both have depth one. Note that this class of TRS is not captured by the HRS of Theorem 1 (when restricted to ranked terms). The above regularity preservation result is no longer true for unranked terms.

Proposition 4. $\mathcal{R}^{*}(L)$ is not a $H A$ language in general when $L$ is a a HA language and $\mathcal{R}$ is a context-free, linear and flat HRS. Moreover, it can be assumed that all the rules of $\mathcal{R}$ are prefix or postfix.

Proof. Let us consider the context-free HRS $\mathcal{R}=\{g(x) \rightarrow g(a x b)\}$ of Example 1, and the HA language $L=\{g(c)\}$. The language $\mathcal{R}^{*}(L)=\left\{g\left(a^{n} c b^{n}\right) \mid n \geq 0\right\}$ is not HA. We can transform the above $\mathcal{R}$ into $\mathcal{R}^{\prime}=\left\{g(x) \rightarrow g^{\prime}(a x), g^{\prime}(y) \rightarrow\right.$ $g(y b)\}$ whose rules are prefix or postfix (and linear) and which is such that $\mathcal{R}^{\prime *}(L) \cap \mathcal{T}(\{g, a, b\})=\mathcal{R}^{*}(L)$.

Note that the language in the above proof is recognized by a CF-HA. We shall show below (Theorem 2 in Section 5) that context-free HRS like the $\mathcal{R}$ above preserve CF-HA languages.

We show now the stronger result that the closure of a HA language under rewriting with a flat HRS, even linear, is neither HA, nor CF-HA and actually not even recursive. 
Proposition 5. $\mathcal{R}^{*}(L)$ is not recursive in general when $L$ is a HA language and $\mathcal{R}$ is a linear and flat HRS whose rules contain at most two variables.

Proof. We reduce the blank accepting problem for TM to ground reachability for an HRS. Let $\mathcal{M}$ be a TM with a tape alphabet $\Gamma$ and a state set $S$ and let $\Sigma=\Gamma \cup S \cup\{g\}$. A configuration of $\mathcal{M}$ is represented by a term $g(w)$ where $w$ is a word of $\Gamma^{*} S \Gamma^{*}$ (the position of the state symbol indicates the position of the head of $\mathcal{M}$ and the rest represents the contents of the tape). We assume, wlog unique blank initial and final configurations, respectively $c_{\mathrm{i}}$ and $c_{\mathrm{f}}$. We consider a HRS $\mathcal{R}$ containing one rule for each transition of $\mathcal{M}$. For instance, $\mathcal{R}$ contains a rule $f(x a s y) \rightarrow f\left(x s^{\prime} a^{\prime} y\right)$ corresponding to a transition $s, a \rightarrow L, s^{\prime}, a^{\prime}$ (with $s, s^{\prime} \in S$ and $\left.a, a^{\prime} \in \Gamma\right)$ and $f(x a s b y) \rightarrow f\left(x a^{\prime} b s^{\prime} y\right)$ to the transition $s, a \rightarrow R, s^{\prime}$. The blank tape is accepted by $\mathcal{M}$ iff $c_{\mathrm{i}} \stackrel{*}{\mathcal{R}} c_{\mathrm{f}}$.

As a consequence, regular hedge model checking is undecidable for the HRS of Proposition 5, according to the remarks in Section 3.2.

\subsection{Rewrite Rules with Flat and One-Variable or Ground Right-Hand-Sides}

If we relax the inverse context-free condition, with only one variable allowed in the rhs of rules, but possibly with two occurrences, both at depth 1 , then the result of Theorem 1, again, is not valid anymore.

Proposition 6. $\mathcal{R}^{*}(L)$ is not recursive in general when $L$ is a HA language and $\mathcal{R}$ is a HRS whose rhs of rules are ground or of the form $d(x x)$.

Proof. We reduce the blank accepting problem for a TM $\mathcal{M}$ with a tape alphabet $\Gamma$ and a state set $S$. Let us consider an alphabet containing all the symbols in $\Gamma, S$ and $f, g, d, d^{\prime}, 0,1,2$ and \#. Like in the proof of Proposition 5, we represent a configuration of $\mathcal{M}$ by a term $g(w)$ with $w \in \Gamma^{*} Q \Gamma^{*}$. We write $c \vdash_{\mathcal{M}} c^{\prime}$ if the configuration $c^{\prime}$ is a successor of $c$ following the transition table of $\mathcal{M}$. It is folklore knowledge that every such pair of configurations has the form $u a_{1} a_{2} a_{3} v \vdash_{\mathcal{M}} u a_{1}^{\prime} a_{2}^{\prime} a_{3}^{\prime} v$ for some $u, v \in \Gamma^{*}$ and $\left(a_{1}, a_{2}, a_{3}, a_{1}^{\prime}, a_{2}^{\prime}, a_{3}^{\prime}\right) \in D_{\mathcal{M}}$, where $D_{\mathcal{M}}$ is a subset of $(\Gamma \cup S)^{6}$ which depends only on $\mathcal{M}$.

A run of $\mathcal{M}$ is a sequence of configuration $c_{0} \vdash_{\mathcal{M}} \ldots \vdash_{\mathcal{M}} c_{n}$, starting with $c_{0}=g\left(q_{\mathrm{i}} b\right)\left(q_{\mathrm{i}}\right.$ is an initial state and $b \in \Gamma$ is the blank symbol), and ending with a final configuration $c_{n}=g\left(u q_{\mathrm{f}} v\right)$ where $q_{\mathrm{f}}$ is a final state and $u, v \in \Gamma^{*}$. We assume wlog that the length of every run is even. A sequence as above is represented as a right comb $f\left(c_{0}, f\left(c_{1}, \ldots f\left(c_{n}, \#\right)\right)\right.$ ) (here $f$ is used as a binary symbol).

The following right-ground rewrite rules reduce a run both to 0 and 1 . The rules reducing to 0 check, for each $1 \leq i \leq \frac{n}{2}$ that $c_{2 i-1} \vdash_{\mathcal{M}} c_{2 i}$, and the rules reducing to 1 check, for each $1 \leq i \leq \frac{n}{2}$ that $c_{2 i} \vdash_{\mathcal{M}} c_{2 i+1}$, and moreover they check the initial and final configurations $c_{0}$ and $c_{n}$.

$$
\begin{aligned}
f\left(g\left(x a_{1} a_{2} a_{3} y\right) f\left(g\left(x a_{1}^{\prime} a_{2}^{\prime} a_{3}^{\prime} y\right) \#\right)\right) \rightarrow 0 & \\
f\left(g\left(x a_{1} a_{2} a_{3} y\right) f\left(g\left(x a_{1}^{\prime} a_{2}^{\prime} a_{3}^{\prime} y\right) 0\right)\right) \rightarrow 0 & \left.f\left(g\left(x q^{\mathrm{f}} y\right) \#\right)\right) \rightarrow 1 \\
f\left(g\left(x a_{1} a_{2} a_{3} y\right) f\left(g\left(x a_{1}^{\prime} a_{2}^{\prime} a_{3}^{\prime} y\right) 1\right)\right) \rightarrow 1 & \left.f\left(g\left(q_{\text {init }} b\right) 1\right)\right) \rightarrow 2
\end{aligned}
$$


We consider also right-ground rewrite rules corresponding to the production rules of a regular tree grammar $\mathcal{G}$, with axiom (initial non-terminal) $I$, which generates right-combs of the above form which are expected to be a run, and a copy $\mathcal{G}^{\prime}$ of $\mathcal{G}$, with a disjoint set of non-terminals, and axiom $I^{\prime}$. Let $\mathcal{R}$ be the set of all the above right-ground rules and the one-variable rule $d(x x) \rightarrow d^{\prime}(x x)$. We have that $d\left(I I^{\prime}\right) \stackrel{*}{\mathcal{R}} d^{\prime}(02)$ iff there exists a run of $\mathcal{M}$ starting with $c_{0}$.

\section{Closure of Context-Free Hedge Automata Languages}

It has been observed [8] that in several cases, one class of word rewrite system preserves regularity and its symmetric class preserves context-free languages. In this section, we prove a similar result by showing that a restricted case of contextfree HRS, i.e. of the symmetric version of the systems considered in Section 4, preserve CF-HA languages. We give next some counterexamples showing that the restrictions are necessary for this result.

\subsection{Linear Restricted Context-Free Rewrite Rules}

We call a HRS $\mathcal{R}$ restricted context-free if it is context-free, and moreover, for all rule $f(x) \rightarrow r \in \mathcal{R}, x$ can occurs in $r$ only at depth at most 1 . Note that this definition includes the case of collapsing rules $f(x) \rightarrow x$.

Theorem 2. The closure $\mathcal{R}^{*}(L)$ of a CF-HA language $L$ under rewriting by a linear restricted context-free $H R S \mathcal{R}$ is a CF-HA language.

Proof. Let $\mathcal{A}_{L}=\left(Q_{L}, Q_{L}^{\mathrm{f}}, \Delta_{L}\right)$ be a normalized CF-HA recognizing $L$. We shall construct an extended CF-HA $\mathcal{A}^{\prime}$ with collapsing transitions (see Section 3.1 for the definition) recognizing $\mathcal{R}^{*}(L)$. The result follows then from Proposition 1 .

First, let us construct for each rule $f(x) \rightarrow g\left(r_{1} \ldots r_{n}\right) \in \mathcal{R}$ and every subterm $r \neq x$ amongst $r_{1}, \ldots, r_{n}$ (let us denote $r h s(\mathcal{R})$ the set of such subterms) a CF-HA (with collapsing transitions) $\mathcal{A}_{r}=\left(Q_{r}, \emptyset, \Delta_{r}\right)$ characterizing the set of ground instances of $r$. We have in $\mathcal{A}_{r}$ one state $q_{u} \in Q_{r}$ for each non-variable subterm $u$ of $r$, and a universal state $q_{\forall} \in Q_{r}$. Below, for every subterm $u$ of $r$, we shall write $q_{u}$ to denote either the state $q_{u}$ if $u$ is not a variable or $q_{\forall}$ otherwise. The set of final states of $\mathcal{A}_{r}$ is left unspecified. It is indeed not relevant to our purpose since $\mathcal{A}_{r}$ is only used as a part of the CF-HA $\mathcal{A}^{\prime}$ constructed below. The transition set $\Delta_{r}$ contains one rule $f\left(q_{u_{1}} \ldots q_{u_{n}}\right) \rightarrow q_{f\left(u_{1} \ldots u_{n}\right)}$ for each subterm $f\left(u_{1} \ldots u_{n}\right)$ of $r$ (as specified above, $q_{i}$ is $q_{\forall}$ if $u_{i}$ is a variable and $q_{i}$ is a state $q_{u_{i}}$ otherwise). It contains moreover one collapsing transition $q_{\forall}^{*} \rightarrow q_{\forall}$ and one transition rule $f\left(q_{\forall}^{*}\right) \rightarrow q_{\forall}$ for each $f \in \Sigma$. The states sets $Q_{r}$ and $Q_{L}$ are assumed pairwise disjoint. Let $\mathcal{A}:=\left(Q, Q_{L}^{\mathrm{f}}, \Delta\right)$ with

$$
Q:=Q_{L} \uplus \biguplus_{r \in r h s(\mathcal{R})} Q_{r} \text { and } \Delta:=\Delta_{L} \uplus \biguplus_{r \in r h s(\mathcal{R})} \Delta_{r} \text {. }
$$

For each $f \in \Sigma, q \in Q$, let $L_{f, q}$ be the context-free language in the transition (assumed unique) $f\left(L_{f, q}\right) \rightarrow q \in \Delta$, and let $\mathcal{G}_{f, q}=\left(Q, N_{f, q}, I_{f, q}, P_{f, q}\right)$ be a CF 
grammar generating $L_{f, q}$, with alphabet (set of terminal symbols) $Q$, set of non terminal symbols $N_{f, q}$, axiom $I_{f, q} \in N_{f, q}$, and set of production rules $P_{f, q}$. The sets of non-terminals $N_{f, q}$ are assumed pairwise disjoint.

We complete the grammars $\mathcal{G}_{f, q}$ with new non-terminals $I_{f, q}^{\prime}$ and some sets $P_{f, q}^{\prime}$ of new production rules containing:

i. $I_{f, q}^{\prime}:=I_{f, q}$ for all $f \in \Sigma, q \in Q$,

ii. $I_{g, q}^{\prime, q}:=q_{r_{1}} \ldots q_{r_{n}} I_{f, q}^{\prime} q_{s_{1}} \ldots q_{s_{m}}$ for each rule $f(x) \rightarrow g\left(r_{1} \ldots r_{n} x s_{1} \ldots s_{m}\right) \in$ $\mathcal{R}$, with $n, m \geq 0$, and $x \notin \operatorname{var}\left(r_{1}, \ldots, r_{n}, s_{1}, \ldots, s_{m}\right)$, and

iii. $I_{g, q}^{\prime}:=q_{r_{1}} \ldots q_{r_{n}}$ (with $n>0$ ), or $I_{g, q}^{\prime}:=\varepsilon$ (with $n=0$ ), for each rule $f(x) \rightarrow g\left(r_{1} \ldots r_{n}\right) \in \mathcal{R}$ with $x \notin \operatorname{var}\left(r_{1}, \ldots, r_{n}\right)$, if $L(\mathcal{A}, q) \cap f(\mathcal{H}(\Sigma)) \neq \emptyset$.

Note that in the cases $i i$ and $i$ ii cover all the cases of linear restricted context-free rewrite rules, except the collapsing rules.

Let $N=\bigcup_{f \in \Sigma, q \in Q}\left(N_{f, q} \cup\left\{I_{f, q}^{\prime}\right\}\right)$ and $P=\bigcup_{f \in \Sigma, q \in Q}\left(P_{f, q} \cup P_{f, q}^{\prime}\right)$.

Let us clean up these sets: if the language generated by a CF grammar $\left(Q, N, I_{f, q}^{\prime}, P\right)$ is empty then we remove $I_{f, q}^{\prime}$ from $N$ and all the productions of $P$ which contain $I_{f, q}^{\prime}$. We iterate this operation, until there is no remaining non-terminals generating an empty language in $N$ (note that the construction stops since we only remove non-terminals and productions). Let us note $N^{\prime}$ and $P^{\prime}$ the sets of non-terminals and productions obtained. For each $f \in \Sigma, q \in Q$, let $\mathcal{G}_{f, q}^{\prime}=\left(Q, N^{\prime}, I_{f, q}^{\prime}, P^{\prime}\right)$, and let $L_{f, q}^{\prime}$ be its language.

Finally, $\mathcal{A}^{\prime}=\left(Q, Q_{L}^{\mathrm{f}}, \Delta^{\prime}\right)$ is obtained by the addition of collapsing transitions corresponding to the collapsing rewrite rules in $\mathcal{R}$

$$
\Delta^{\prime}=\left\{f\left(L_{f, q}^{\prime}\right) \rightarrow q \mid f \in \Sigma, q \in Q, L_{f, q}^{\prime} \neq \emptyset\right\} \cup\left\{L_{f, q}^{\prime} \rightarrow q \mid f(x) \rightarrow x \in \mathcal{R}\right\}
$$

We show that $L\left(\mathcal{A}^{\prime}\right)=\mathcal{R}^{*}(L(\mathcal{A}))$.

Direction $L\left(\mathcal{A}^{\prime}\right) \subseteq \mathcal{R}^{*}(L(\mathcal{A}))$. We show more generally that for all $t \in L\left(\mathcal{A}^{\prime}, q_{0}\right)$, $q_{0} \in Q$, there exists $u \in L\left(\mathcal{A}, q_{0}\right)$ such that $u \stackrel{*}{\mathcal{R}} t$. The proof is by induction on the number of applications of collapsing transitions other than $q_{\forall}^{*} \rightarrow q_{\forall}$ in the reduction $t \underset{\mathcal{A}^{\prime}}{\stackrel{*}{a}} q_{0}$.

Induction step. Let us prove first the induction step (we shall prove the base case later). Assume that the reduction $t \stackrel{*}{\mathcal{A}^{\prime}} q_{0}$ has the form

$$
t=C[h] \stackrel{*}{\stackrel{\mathcal{A}^{\prime}}{\prime}} C\left[q_{1} \ldots q_{n}\right] \underset{\mathcal{A}^{\prime}}{\longrightarrow} C[q] \stackrel{*}{\stackrel{\mathcal{A}^{\prime}}{\rightarrow}} q_{0}
$$

and that there exists a collapsing transition $L_{f, q}^{\prime} \rightarrow q$ with $q_{1} \ldots q_{n} \in L_{f, q}^{\prime}$. By construction it means that $\mathcal{R}$ contains a collapsing rule $f(x) \rightarrow x$. We have then

$$
s=C[f(h)] \stackrel{*}{\mathcal{A}^{\prime}} C\left[f\left(q_{1} \ldots q_{n}\right)\right] \underset{\mathcal{A}^{\prime}}{\longrightarrow} C[q] \stackrel{*}{\underset{\mathcal{A}^{\prime}}{\longrightarrow}} q_{0}
$$

The reduction $s \underset{\mathcal{A}^{\prime}}{\stackrel{*}{\mathcal{A}^{\prime}}} q_{0}$ applies one collapsing transition less than $t \underset{\mathcal{A}_{*}^{\prime}}{\stackrel{*}{\mathcal{A}^{\prime}}} q_{0}$. Hence, by induction hypothesis, there exists $u \in L\left(\mathcal{A}, q_{0}\right)$ such that $u \stackrel{*}{\mathcal{R}} s$. Moreover, $u \stackrel{*}{\mathcal{R}} t$ because $s \underset{\mathcal{R}}{\rightarrow} t$ with the rule $f(x) \rightarrow x$. 
Base case. Now for the base case (no collapsing transition applied, except $q_{\forall}^{*} \rightarrow q_{\forall}$ ), we make a second induction on the number of occurrences of nonterminals of the form $I_{f, p}^{\prime}$ in all the derivations, by the grammars $\mathcal{G}_{f, p}^{\prime}$, of the sequences of states $q_{1} \ldots q_{n} \in Q^{*}$ used in transitions of the form $f\left(q_{1} \ldots q_{n}\right) \rightarrow q$ in the reduction $t \stackrel{*}{\mathcal{A}^{\prime}} q_{0}$. Let us note $\vdash$ the relation of derivation using the rules of $P^{\prime}$, and $\vdash^{*}$ its transitive closure. More precisely, we consider the number of occurrences of non terminals $I_{f, p}^{\prime}$ occurring in derivations of the form $I_{g, q}^{\prime} \vdash q_{1} \ldots q_{n}$, excluding the first occurrence of $I_{g, q}^{\prime}$ (at the beginning of the derivation). Intuitively every such $I_{f, p}^{\prime}$ corresponds to a rewrite step with a context-free rule of $\mathcal{R}$ in the rewrite sequence $u \stackrel{*}{\mathcal{R}} t$.

Base case (second induction). For the base case, the number occurrences of nonterminals of the form $I_{g, q}^{\prime}$ is zero. It means that $t \underset{\mathcal{A}}{\stackrel{*}{\longrightarrow}} q_{0}$ (every derivation starts by $I_{g, q}^{\prime} \vdash I_{g, q}$, case $i$ of the above construction), and we let $u=t$.

Induction step (second induction). Assume that the reduction $t \underset{\mathcal{A}^{\prime}}{\rightarrow} q_{0}$ has the form

$$
t=C\left[g\left(t_{1} \ldots t_{k}\right)\right] \underset{\mathcal{A}^{\prime}}{\stackrel{*}{\longrightarrow}} C\left[g\left(q_{1} \ldots q_{k}\right)\right] \underset{\mathcal{A}^{\prime}}{\longrightarrow} C[q] \stackrel{*}{\underset{\mathcal{A}^{\prime}}{\longrightarrow}} q_{0}
$$

where $g\left(q_{1} \ldots q_{k}\right) \underset{\mathcal{A}^{\prime}}{\longrightarrow} q$ is the first transition such that the derivation of $I_{g, q}^{\prime} \vdash^{*}$ $q_{1} \ldots q_{k}$ by $\mathcal{G}_{g, q}^{\prime}$ involves one non-terminal of the form $I_{f, q^{\prime}}^{\prime}$ (other than the first occurrence of $I_{g, q}^{\prime}$ at the beginning of the derivation).

Note that we can assume that for every $i \leq k, t_{i} \underset{\mathcal{A}^{\prime}}{*} q_{i}$ because no collapsing transition are used by hypothesis. Hence, together with the above hypothesis, it implies that $t_{i} \in L\left(\mathcal{A}, q_{i}\right)$ for all $i \leq k$.

By construction, the derivation $I_{g, q}^{\prime} \vdash^{*} q_{1} \ldots q_{k}$ starts with a production rule of $P^{\prime}$ added by one of the cases $i$ or iii above.

Case ii. We have $I_{g, q}^{\prime} \vdash q_{r_{1}} \ldots q_{r_{n}} I_{f, q}^{\prime} q_{s_{1}} \ldots q_{s_{m}} \vdash^{*} q_{1} \ldots q_{k}$, and the first production rule used in this derivation was added by the case $i i$ of the construction because there exists a rewrite rule $g(x) \rightarrow f\left(r_{1} \ldots r_{n} x s_{1} \ldots s_{m}\right) \in \mathcal{R}$. It follows that $I_{f, q}^{\prime} \vdash^{*} q_{n+1} \ldots q_{n+p}$, with $p=k-m-n$, and that

$$
s=C\left[f\left(t_{n+1} \ldots t_{n+p}\right)\right] \underset{\mathcal{A}^{\prime}}{\stackrel{*}{\longrightarrow}} C\left[f\left(q_{n+1} \ldots q_{n+p}\right)\right] \underset{\mathcal{A}^{\prime}}{\longrightarrow} C[q] \stackrel{*}{\underset{\mathcal{A}^{\prime}}{\longrightarrow}} q_{0} .
$$

The above reduction sequence is strictly smaller than the above $t \stackrel{*}{\mathcal{A}^{\prime}} q_{0}$ wrt the number of non-terminals of the form $I^{\prime}$ used in derivations. Hence, by induction hypothesis, there exists $u \in L\left(\mathcal{A}, q_{0}\right)$ such that $u \stackrel{*}{\mathcal{R}} s$.

Moreover, $q_{1}=q_{r_{1}}, \ldots, q_{n}=q_{r_{n}}$ and $q_{n+p+1}=q_{s_{1}}, \ldots, q_{k}=q_{s_{m}}$. Recall that by convention, the $q_{r_{i}}$ and $q_{s_{i}}$ can denote either a state $q_{u}$ associated to a non-variable subterm $u$ of a right member of rule of $\mathcal{R}$ or $q_{\forall}$. Therefore, since $t_{i} \in$ $L\left(\mathcal{A}, q_{i}\right)$ for all $i \leq k, t_{1}$ is an instance of $r_{1}, \ldots, t_{n}$ is an instance of $r_{n}, t_{n+p+1}$ is an instance of $s_{1}, \ldots, t_{k}$ is an instance of $s_{m}$. Therefore $f\left(t_{n+1} \ldots t_{n+p}\right) \underset{\mathcal{R}}{\longrightarrow}$ $g\left(t_{1} \ldots t_{k}\right)$, and $u \stackrel{*}{\stackrel{*}{\mathcal{R}}} t$. 
Case iii. We have $I_{g, q}^{\prime} \vdash q_{1} \ldots q_{k}$ and the production rule used was added by the case $i i i$ of the construction of $P^{\prime}$ because there is a rewrite rule $f(x) \rightarrow$ $g\left(r_{1} \ldots r_{k}\right) \in \mathcal{R}$, with $x \notin \operatorname{var}\left(r_{1}, \ldots, r_{n}\right)$, and for all $i \leq k, q_{i}=q_{r_{i}}$ (again, it can denote $q_{\forall}$ ). Hence, for all $i \leq k, t_{i}$ is an instance of $r_{i}$ by hypotheses. Because of the condition in the case $i i i$ of the construction, there exists $h \in \mathcal{H}(\Sigma)$ such that $f(h) \in L(\mathcal{A}, q)$. Hence $s=C[f(h)] \underset{\mathcal{A}^{\prime}}{*} C[q] \underset{\mathcal{A}^{\prime}}{\stackrel{*}{\mathcal{A}^{\prime}}} q_{0}$. By induction hypothesis, there exists $u \in L\left(\mathcal{A}, q_{0}\right)$ such that $u \stackrel{\mathcal{A}^{\prime}}{\mathcal{R}} s$, and $u \stackrel{*}{\mathcal{R}} t$ because $s \underset{\mathcal{R}}{\longrightarrow} t$.

Direction $L\left(\mathcal{A}^{\prime}\right) \supseteq \mathcal{R}^{*}(L(\mathcal{A}))$. We show that for all $u \in L(\mathcal{A})$, if $u \stackrel{*}{\mathcal{R}} t$, then $t \in L\left(\mathcal{A}^{\prime}\right)$, by induction on the length of the rewrite sequence.

Base case (0 rewrite steps). In this case, $u=t \in L(\mathcal{A})$. We can note that $L(\mathcal{A}) \subseteq L\left(\mathcal{A}^{\prime}\right)$, because of the productions $I_{f, q}^{\prime}:=I_{f, q}$ added by the case $i$ of the construction. Hence, $t \in L\left(\mathcal{A}^{\prime}\right)$.

Induction step $(k+1$ rewrite steps). We have three cases.

Case $i$. The last rewrite step of the sequence involves a collapsing rewrite rule $f(x) \rightarrow x \in \mathcal{R}:$

$$
u \stackrel{*}{\stackrel{\mathcal{R}}{\longrightarrow}} C[f(h)] \underset{\mathcal{R}}{\longrightarrow} C[h]=t .
$$

By induction hypothesis, $C[f(h)] \in L\left(\mathcal{A}^{\prime}\right)$. Hence there exists a reduction sequence: $C[f(h)] \stackrel{*}{\mathcal{A}^{\prime}} C\left[f\left(q_{1} \ldots q_{n}\right)\right] \underset{\mathcal{A}^{\prime}}{\stackrel{(}{\mathcal{A}^{\prime}}} C[q] \stackrel{*}{\mathcal{A}^{\prime}} q_{\mathrm{f}} \in Q_{L}^{\mathrm{f}}$ with $q_{1} \ldots q_{n} \in L_{f, q}^{\prime}$. By construction, $\mathcal{A}^{\prime}$ contains a collapsing transition rule $L_{f, q}^{\prime} \rightarrow q$. Hence $t=$ $C[h] \underset{\mathcal{A}^{\prime}}{\stackrel{*}{\longrightarrow}} C\left[q_{1} \ldots q_{n}\right] \underset{\mathcal{A}^{\prime}}{\longrightarrow} C[q] \underset{\mathcal{A}^{\prime}}{\stackrel{*}{\longrightarrow}} q_{\mathrm{f}} \in Q_{L}^{\mathrm{f}}$, i.e. $t \in L\left(\mathcal{A}^{\prime}\right)$.

Case ii. The last rewrite step of the sequence involves a context-free rewrite rule $f(x) \rightarrow g\left(r_{1} \ldots r_{n} x s_{1} \ldots s_{m}\right) \in \mathcal{R}$, with $n, m \geq 0$

$$
u \underset{\mathcal{R}}{\stackrel{*}{\longrightarrow}} C[f(h)] \underset{\mathcal{R}}{\longrightarrow} C\left[g\left(r_{1} \ldots r_{n} h s_{1} \ldots s_{m}\right) \sigma\right]=t .
$$

By induction hypothesis, $C[f(h)] \in L\left(\mathcal{A}^{\prime}\right)$, hence, there exists a reduction sequence: $C[f(h)] \stackrel{*}{\mathcal{A}^{\prime}} C\left[f\left(q_{1} \ldots q_{p}\right)\right] \underset{\mathcal{A}^{\prime}}{\longrightarrow} C[q] \stackrel{*}{\mathcal{A}^{\prime}} q_{\mathrm{f}} \in Q_{L}^{\mathrm{f}}$ with $q_{1} \ldots q_{p} \in L_{f, q}^{\prime}$, i.e. $I_{f, q}^{\prime} \vdash^{*} q_{1} \ldots q_{p}$. By the case $i i$ of the construction, we have $I_{g, q}^{\prime}:=$ $q_{r_{1}} \ldots q_{r_{n}} I_{f, q}^{\prime} q_{s_{1}} \ldots q_{s_{m}} \in P^{\prime}$ (note that both $I_{g, q}^{\prime}$ and $I_{f, q}^{\prime}$ are clean, i.e. members of $\left.N^{\prime}\right)$, hence $q_{r_{1}} \ldots q_{r_{n}} q_{1} \ldots q_{p} q_{s_{1}} \ldots q_{s_{m}} \in L_{g, q}^{\prime}$. If follows that $t \frac{*}{\mathcal{A}^{\prime}}$ $C\left[g\left(q_{r_{1}} \ldots q_{r_{n}} q_{1} \ldots q_{p} q_{s_{1}} \ldots q_{s_{m}}\right)\right] \underset{\mathcal{A}^{\prime}}{\longrightarrow} C[q] \underset{\mathcal{A}^{\prime}}{*} q_{\mathrm{f}}$, i.e. $t \in L\left(\mathcal{A}^{\prime}\right)$.

Case iii. The last rewrite step of the sequence involves a context-free rewrite rule $f(x) \rightarrow g\left(r_{1} \ldots r_{n}\right) \in \mathcal{R}$ with $x \notin \operatorname{var}\left(r_{1} \ldots r_{n}\right)$

$$
u \stackrel{*}{\mathcal{R}} C[f(h)] \underset{\mathcal{R}}{\longrightarrow} C\left[g\left(r_{1} \ldots r_{n}\right) \sigma\right]=t .
$$

By induction hypothesis, $f(h) \in L\left(\mathcal{A}^{\prime}\right)$. With the case $i i$ of the construction, $I_{g, q}^{\prime}:=q_{r_{1}} \ldots q_{r_{n}} \in P^{\prime}$, hence $q_{r_{1}} \ldots q_{r_{n}} \in L_{g, q}^{\prime}$. Since for all $i \leq n, r_{i} \sigma \underset{\mathcal{A}^{\prime}}{*} q_{r_{i}}$, $t \in L\left(\mathcal{A}^{\prime}, q\right)$. (end of the proof of Theorem 2) 
Corollary 2. Reachability and regular hedge model-checking are decidable for linear restricted context-free HRS.

Proof. The intersection of an CF-HA language and a HA languages is a CF-HA language, and emptiness of CF-HA is decidable.

It is shown in [17] that the languages of CF-HA are closures of regular tree languages modulo associativity of one or several binary function symbols. Therefore, the above results are also valid for these languages.

\subsection{Linear Context-Free Rewrite Rules}

Context-free HRS are named after context-free tree grammars, whose production rules have the form $N\left(x_{1}, \ldots, x_{n}\right) \rightarrow r$ where $N$ is a non-terminal of arity $n$ (from a finite set $\mathcal{N}), x_{1}, \ldots, x_{n} \in \mathcal{X}$ and $r \in \mathcal{T}\left(\Sigma \cup \mathcal{N},\left\{x_{1}, \ldots, x_{n}\right\}\right)$. Note that our definition of context-free HRS is restricted to unary non-terminals. However, even for this case of unary non-terminals and right-linear rewrite rules, the result of Theorem 2 cannot be generalized to context-free HRS.

Proposition 7. $\mathcal{R}^{*}(L)$ is not a CF-HA language in general when $L$ is a CF-HA language and $\mathcal{R}$ is a linear context-free $H R S$.

Proof. Let us consider the context-free HRS: $\mathcal{R}=\{f(x) \rightarrow g(f(a x))\}$ and let $L=\{f(c)\}$. The set $\mathcal{R}^{*}(L)$ is $\left.\left.\{\underbrace{g(g(\ldots g}_{n}\left(f\left(a^{n} c\right)\right))\right) \mid n \in \mathbb{N}\right\}$.

Using a pumping argument, we can show that it is not a CF-HA language. Assume indeed that it is recognized by a CF-HA $\mathcal{A}$ with state set $Q$. In a term of $\mathcal{R}^{*}(L)$ with $n>|Q|$, there will two subterms $u=g^{i}\left(f\left(a^{n} c\right)\right)$ and $v=g^{j}(u)$, with $i, j>0$, both in $L(\mathcal{A}, q)$ for some $q \in Q$. The term $g^{n-j}\left(f\left(a^{n} c\right)\right)$ is recognized by $\mathcal{A}$, and is not in $\mathcal{R}^{*}(L)$.

The above counter-example shows the importance for Theorem 2 of the condition, in the definition of restricted context-free HRS, that the variable $x$ in a lhs of rule occurs at a shallow position in the corresponding rhs.

\subsection{Restricted Context-Free Rewrite Rules}

If we keep the restricted context-free condition (the variable $x$ in the lhs of a rule occurs at a shallow position in the corresponding rhs) but we drop the linearity condition, we also lose the CF-HA preservation result of Theorem 2.

Proposition 8. $\mathcal{R}^{*}(L)$ is not a CF-HA language in general when $L$ is a CF-HA language and $\mathcal{R}$ is a restricted context-free HRS.

Proof. Let $\mathcal{R}=\{f(x) \rightarrow f(x x)\}$ and $L=\{f(a)\}$. We have that $\mathcal{R}^{*}(L)=$ $\left\{f\left(a^{n}\right) \mid n=2^{k}, k \geq 0\right\}$ which is not a CF-HA language. Assume indeed that this language is recognized by a CF-HA $\left(Q, Q^{\mathrm{f}}, \Delta\right)$. It means that $\Delta$ contains a transition $f(L) \rightarrow q$ where $L$ is a context-free language of words of $Q^{*}$ of length 
$2^{k}, k \geq 0$. The image of $L$ under the strictly alphabetic homomorphism which translates every state $q \in Q$ into $a$ is context-free. As it is a one letter language, it is also regular. But it is well known that this language $\left\{a^{n} \mid n=2^{k}, k \geq 0\right\}$ is actually not regular.

\subsection{Mixing Inverse CF and Restricted CF Rewrite Rules}

We show now that the results of Theorems 1 and 2 cannot be combined. In other terms, for some HRS containing both linear inverse context-free and restricted context-free rules, the set of descendants of a HA language is not a HA language, neither a CF-HA language and even not recursive.

Proposition 9. $\mathcal{R}^{*}(L)$ is not recursive in general when $L$ is a HA language and $\mathcal{R}$ is a HRS whose rules are either inverse context-free or restricted context-free and contain only one variable.

Proof. We reduce the Post Correspondence Problem (PCP). Let us consider an instance $\mathcal{P}=\left\{\left\langle u_{i}, v_{i}\right\rangle \mid i \leq n, u_{i}, v_{i} \in \Gamma^{*}\right\}$ of PCP on an finite alphabet $\Gamma$. The problem is to find a sequence $i_{1}, \ldots, i_{k} \leq n$ such that $u_{i_{1}} \ldots u_{i_{k}}=v_{i_{1}} \ldots v_{i_{k}}$

Let $\mathcal{R}$ be an HRS containing a rule $f_{0}(x) \rightarrow f_{0}\left(\widetilde{u}_{i} x v_{i}\right)$ for each pair $\left\langle u_{i}, v_{i}\right\rangle \in$ $\mathcal{P}\left(\tilde{u_{i}}\right.$ is the mirror image of $\left.u_{i}\right)$, and two rules $f_{0}(a x a) \rightarrow f_{1}(x)$ and $f_{1}(a x a) \rightarrow$ $f_{1}(x)$ for each $a \in \Gamma$. We assume that $f_{0}, f_{1}$, and $c$ are symbols not in $\Gamma$. We have that $f_{0}(c) \stackrel{*}{\mathcal{R}} f_{1}(c)$ iff $\mathcal{P}$ has a solution.

Moreover, as we have shown that context-free HRS do not preserve HA languages (Proposition 4), the symmetric also holds for inverse-context-free HRS and CF-HA languages.

Proposition 10. $\mathcal{R}^{*}(L)$ is not recursive in general when $L$ is a CF-HA language and $\mathcal{R}$ is an inverse context-free $H R S$.

Proof. Let $\mathcal{R}_{1}$ be the subset of the context-free rewrite rules of the HRS of the above proof of Proposition 9 , and $\mathcal{R}_{2}$ be the subset of the other rules. Note that $\mathcal{R}_{2}$ is an inverse context-free HRS.

By Theorem $2, L=\mathcal{R}_{1}^{*}\left(\left\{f_{0}(c)\right\}\right)$ is a CF-HA language. Like in the proof of Proposition 9 , we have that $f_{1}(c) \in \mathcal{R}_{2}^{*}(L)$ iff the PCP has a solution. Hence, because of the decidability of the membership problem for CF-HA, $\mathcal{R}_{2}^{*}(L)$ cannot be a CF-HA language.

\section{Conclusion}

We have shown that HA and CF-HA languages are preserved by rewrite closure for interesting classes of non ground hedge rewriting rules. These rules allow us for instance to modify the structure of XML documents when processing them. We plan to extend our results to non ordered unranked trees by considering sheaves automata as in [5] or commutative hedge automata (see [3] for application to process rewrite systems). 
Regularity preservation has been studied in the case of ranked terms for transducing term rewriting system, i.e. rewrite rules corresponding to transducers rules [20]. A generalization of such classes of TRS to hedge rewriting seems conceptually close to XML transformations [12] and we plan to study the preservation of HA or CF-HA languages w.r.t. to such HRS.

\section{References}

1. P. A. Abdulla, B. Jonsson, M. Nilsson, and M. Saksena. A survey of regular model checking. In Proc. of the 15th Int. Conf. on Concurrency Theory (CONCUR'04), vol. 3170 of $L N C S$, pages 35-48. Springer, 2004.

2. A. Bouajjani, B. Jonsson, M. Nilsson, and T. Touili. Regular model checking. In Proc. of the 12th Int. Conf. on Computer Aided Verification (CAV'00), vol. 1855 of $L N C S$, pages 403-418, 2000.

3. A. Bouajjani and T. Touili. On computing reachability sets of process rewrite systems. In Proc. 16th Int. Conf. Term Rewriting and Applications (RTA'05), vol. 3467 of LNCS, pages 484-499. Springer, 2005.

4. H. Comon, M. Dauchet, R. Gilleron, F. Jacquemard, D. Lugiez, S. Tison, and M. Tommasi. Tree automata techniques and applications. Available on: http://www.grappa.univ-lille3.fr/tata. Last release October, 12th 2007.

5. S. Dal-Zilio and D. Lugiez. XML schema, tree logic and sheaves automata. In Proc. 14th Int. Conf. Rewriting Techniques and Applications (RTA'03), vol. 2706 of $L N C S$, pages 246-263. Springer, 2003.

6. I. Durand and G. Sénizergues. Bottom-up rewriting is inverse recognizability preserving. In Proc. 18th Int. Conf. Term Rewriting and Applications (RTA'07), vol. 4533 of LNCS, pages 107-121. Springer, 2007.

7. R. Gilleron and S. Tison. Regular tree languages and rewrite systems. Fundamenta Informaticae, 24(1/2):157-176, 1995.

8. D. Hofbauer and J. Waldmann. Deleting string rewriting systems preserve regularity. Theor. Comput. Sci., 327(3):301-317, 2004.

9. F. Jacquemard. Decidable approximations of term rewriting systems. In Proc. of the 7th Int. Conf. on Rewriting Techniques and Applications (RTA'96), vol. 1103 of $L N C S$, pages 362-376. Springer Verlag, 1996.

10. F. Jacquemard and M. Rusinowitch. Rewrite closure of hedge-automata languages. Research Report LSV-08-05, Laboratoire Spécification et Vérification, ENS Cachan, France, 2007. Available on: http://www.lsv.ens-cachan.fr/Publis

11. C. Löding and A. Spelten. Transition graphs of rewriting systems over unranked trees. In Proc. 32nd Int. Symposium on Mathematical Foundations of Computer Science (MFCS'07), vol. 4708 of $L N C S$, pages 67-77, 2007.

12. W. Martens and F. Neven. On the complexity of typechecking top-down XML transformations. Theor. Comput. Sci. Vol 336, N. 1, 2005, pages 153-180.

13. M. Murata. "Hedge Automata: a Formal Model for XML Schemata". http://www.horobi.com/Projects/RELAX/Archive/hedge_nice.html, 2000.

14. M. Murata, D. Lee, and M. Mani. Taxonomy of xml schema languages using formal language theory. In In Extreme Markup Languages, 2001.

15. T. Nagaya and Y. Toyama. Decidability for left-linear growing term rewriting systems. In Proc. 10th Int. Conf. on Rewriting Techniques and Applications (RTA'99), vol. 1631 of LNCS, pages 256-270. Springer Verlag, 1999. 
16. H. Ohsaki. Beyond the regularity: Equational tree automata for associative and commutative theories. In Proc. of CSL'01, vol. 2142 of LNCS. Springer, 2001.

17. H. Ohsaki, H. Seki, and T. Takai. Recognizing boolean closed A-tree languages with membership conditional rewriting mechanism. In Proc. of the 14th Int. Conf. on Rewriting Techniques and Applications (RTA'03), vol. 2706 of LNCS, pages 483-498. Springer Verlag, 2003.

18. J. d'Orso and T. Touili. Regular hedge model checking. In Proc. of the 4th IFIP Int. Conf. on Theoretical Computer Science (TCS'06). IFIP, 2006.

19. K. Salomaa. Deterministic Tree Pushdown Automata and Monadic Tree Rewriting Systems. J. of Comp. and System Sci., vol. 37, pages 367-394, 1988.

20. H. Seki, T. Takai, Y. Fujinaka and Y. Kaji. Layered Transducing Term Rewriting System and Its Recognizability Preserving Property. In Proc. of 13th Int. Conf. on Rewriting Techniques and Applications (RTA'02), vol. 2378 of LNCS, pages 98-113, 2002.

21. T. Takai, Y. Kaji, and H. Seki. Right-linear finite path overlapping term rewriting systems effectively preserve recognizability. In Proc. of 11th Int. Conf. on Rewriting Techniques and Applications (RTA'00), vol. 1833 of LNCS, pages 246-260, 2000.

22. T. Touili. Computing transitive closures of hedge transformations. In In Proc. 1 st Int. Workshop on Verification and Evaluation of Computer and Communication Systems (VECOS'07), eWIC Series. British Computer Society, 2007. 